\title{
Pyramidal reaction wheel arrangement optimization of satellite attitude control subsystem for minimizing power consumption
}

\author{
Abolfazl Shirazi* and Mehran Mirshams** \\ Space Research Laboratory, Aerospace Engineering Department, K.N. Toosi University of Technology, Tehran, Iran
}

\begin{abstract}
The pyramidal reaction wheel arrangement is one of the configurations that can be used in attitude control simulators for evaluation of attitude control performance in satellites. In this arrangement, the wheels are oriented in a pyramidal configuration with a tilt angle. In this paper, a study of pyramidal reaction wheel arrangement is carried out in order to find the optimum tilt angle that minimizes total power consumption of the system. The attitude control system is analyzed and the pyramidal configuration is implemented in numerical simulation. Optimization is carried out by using an iterative process and the optimum tilt angle that provides minimum system power consumption is obtained. Simulation results show that the system requires the least power by using optimum tilt angle in reaction wheels arrangement.
\end{abstract}

Key words: Pyramidal Arrangement, Satellite, Attitude Control, Reaction Wheel

\section{Introduction}

The attitude control subsystem is one of the main auxiliary systems in satellites. It is a three axes stabilized platform, allowing the satellite to point and correct in all directions in orbital missions. Its reliability and cost is important for the reliability and cost of the satellite as a whole. To evaluate the attitude control performance, not only numerical simulations, as well as some experiments using real mechanisms have been made, including attitude control simulators supported by spherical air bearings. Such simulators provide a low cost method of simulating satellite dynamics in a torquefree environment. Actuators such as reaction wheels can be implemented in these simulators in different configurations.

The attitude control of small satellites is generally fulfilled by passive, semi-passive, and active systems. Active attitude control systems, like three-rotor reaction wheel systems, three-magnetorquers systems, or their combinations, with actuators enable to vary control torque, have been widely studied in recent years.

If the satellite is equipped with three independent actuators, a complete solution to the set point and tracking control problems is available.

Dai and Jin [1] implemented a PD controller with friction compensation to stabilize the attitude control simulator. This controller produces attitude error of less than $0.02^{\circ}$ on the $\mathrm{X} / \mathrm{Y}$ axes and $0.05^{\circ}$ on the $\mathrm{Z}$ axis. Another 3-axis control approach based on a scissored-pair CMG with parallel gimbal axes is presented by Steyn [2]. In this approach, an actuator is aligned along each principal body axis of the satellite to provide full three-axis control authority and maximize torque capability. Cruz and Bernstein [3] applied retrospective cost adaptive control to satellite attitude control using reaction-wheel actuators. This approach resulted acceptable accuracies for motion-to-rest maneuvers with unknown constant disturbances. Wisniewski and Kulczycki [4] also proposed the slew maneuver controller for a spacecraft equipped with a star camera and four reaction wheels in the tetrahedron configuration that can be used for on-board implementation. Wang et al. [5] showed that using the variable structure controller to substitute conventional PID can decrease the influence of reaction wheel static friction and improve attitude performance.

Besides the importance of controller design, the arrangement
This is an Open Access article distributed under the terms of the Creative Commons Attribution Non-Commercial License (http://creativecommons.org/licenses/bync/3.0/) which permits unrestricted non-commercial use, distribution, and reproduction in any medium, provided the original work is properly cited. (cc) * Master of Science, Corresponding author : ashirazi@mail.kntu.ac.ir ** Associate Professor 
of actuators has become a significant issue in many studies. Pyramidal configuration is an approach that can be considered as a specific arrangement of actuators in satellites and attitude simulators. Ismail and Varatharajoo [6] investigated several arrangements of actuators including three and four reaction wheels without questioning the effect of tilt angle changes in system performance. Guo et al. [7] presented a simple steering law for spacecraft attitude maneuver with control moment gyroscopes as an actuator in a pyramidal arrangement. However, no discussion is made about pyramidal arrangement optimization; the tilt angle was assumed to be $53.1^{\circ}$. Haruhisa Kurokawa [8] showed that the pyramid type single gimbal CMG system is one of the most effective candidate torquer for attitude control, having such advantages as a simple mechanism, a simpler steering law, and a larger angular momentum space. In that study, it was concluded that if the skew angle of pyramidal arrangement is $\tan ^{-1}(1 / 2)$, then the size of the workspace (allowed region of the angular momentum vector) along the three axes is almost identical. This configuration therefore gives the maximum unidirectional workspace size. If a spherical workspace is desired for convenience of the attitude control, this is the best configuration of four unit systems. Moreover, he showed that there is an optimal skew angle giving the largest workspace for each value of the aspect ratio in pyramidal systems. California Polytechnic State University has been developing the Cal Poly Spacecraft Attitude Dynamics Simulator (SADS), a reaction wheel controlled air bearing simulator. One of the design goals of the SADS project is to be able to easily test and verify spacecraft attitude control laws. Downs [9] and Silva [10] stated that the four reaction wheels are angled with the spin axis inclined by 57 to 61 degrees in this simulator. Also, Logan [11] stated that although the optimum configuration for equal tri-directional control is 53 degrees, the higher angle value allowed for more $\mathrm{z}$-axis control authority which assisted in holding the platform upright.

According to previous researches, it can be seen that great attention has been paid to design the control laws of attitude control system without consideration of the arrangement optimization of reaction wheels. In some researches the value of tilt angle is mentioned but the optimality of this parameter for having minimum power consumption was not the main objective of the researches. Therefore, the question of which tilt angle provides minimum power consumption remains unanswered.

In this paper the problem of attitude controlbyoptimization of pyramidal reaction wheel arrangement based on changes in tilt angle is considered. The performance of such systems is simulated and the total power consumption of system is determined with different tilt angles. The variation of pyramidal reaction wheel arrangement along with changes in tilt angle is analyzed and the optimum tilt angle that optimizes the power consumption is obtained based on the simulation. As our simulation shows, the pyramidal reaction wheel arrangement with optimum tilt angle has an appropriate accuracy and does not need powerful sources of energy and therefore can be used for attitude control of satellites.

The paper is organized as follows. In section 2 the statement of the problem is given. Section 3 is devoted to the dynamics of attitude simulators. Simulation of the performance of the attitude control simulators with pyramidal reaction wheel arrangement is discussed in sections 4 . Finally, the optimization of tilt angle in pyramidal arrangement is carried out in section 5 considering the simulations in previous section. Conclusions and discussion are given in Section 6 .

\section{Statement of the Problem}

Configuration with redundant actuators is often adopted to ensure that the attitude control system can generate any direction of torque even if some of the actuators fail to work.

Consider an attitude control simulator with four reaction wheels in a pyramidal arrangement as depicted in Fig. 1.

Fig. 1 shows the reference frame configuration which will be referred to throughout this paper. In this type of arrangement, the reaction wheels are aligned with the $+x,+y,-x$ and $-y$ body axes and inclined by the angle $\beta$ as depicted in Fig. 2 .
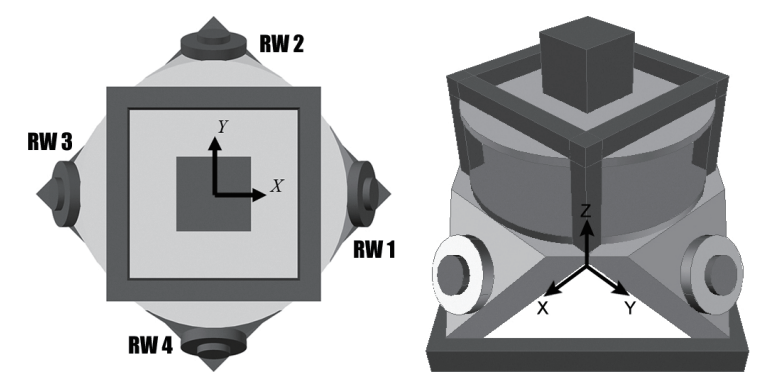

Fig. 1. Reaction wheels arrangement in pyramidal configuration

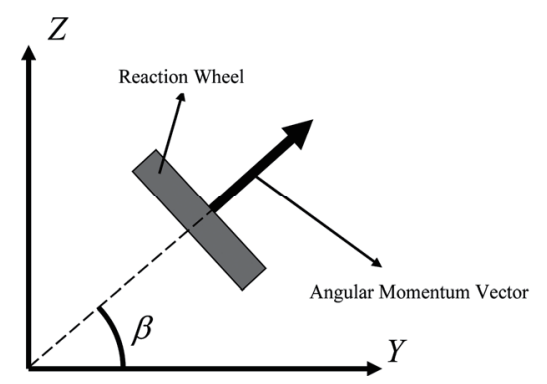

Fig. 2. Tilt angle in pyramidal configuration 
The angle $\beta$ (or its complementary angle) is known by many different names such as "tilt angle" [6], "skew angle" [8], "inclination angle" [10], or "scissor angle" [12]. This concept has been extended to enhance control authority and saturation times, while additionally adding redundancy to the system.

A simple estimation of system performance can be made at first sight. If the tilt angle is assumed to be 0 or 90 degrees, the control components in either one of the $\mathrm{X}, \mathrm{Y}$, or $\mathrm{Z}$ axis will be equal to zero and therefore three axis control will not be applied to the system.

As this angle approaches zero, the control component on the $\mathrm{X}$ and $\mathrm{Y}$ axis becomes larger. On the other hand, more control torques will be supplied for the $\mathrm{Z}$ axis by choosing a high tilt angle. It implies that there is an optimum tilt angle which results in the best efficiency of the system. In the design process of such systems, choosing a proper tilt angle is an important issue. The problem that discussed in this paper is finding the optimum tilt angle, which depends on the desired maneuverability of the system. One of the design criteria of this system is minimizing the total power consumption of the system during the attitude maneuver. Obviously, choosing a proper tilt angle will lead to achieve minimum power consumption. Also, other criteria such as maximum rotational speed of the wheels can be assumed as the main goal in this optimization as the availability of bearing in reaction wheel manufacturing process limits the maximum rotational speed of the wheels.

\section{Modeling the Attitude Control System}

The well-known Euler's moment equation is used in modeling the attitude control system. Three scalar equations which describe Euler's moment equation can be written as follows $[13,14]$.

$$
\begin{aligned}
& M_{x}=I_{x} \dot{\omega}_{x}+\omega_{y} \omega_{z}\left(I_{z}-I_{y}\right) \\
& M_{y}=I_{y} \dot{\omega}_{y}+\omega_{x} \omega_{z}\left(I_{x}-I_{z}\right) \\
& M_{z}=I_{z} \dot{\omega}_{z}+\omega_{x} \omega_{y}\left(I_{y}-I_{x}\right)
\end{aligned}
$$

Where $M_{x}, M_{y}, M_{z}$ are the torques applied on each axis, $\omega_{x}, \omega_{y}, \omega_{z}$ are the angular velocitiy components around each axes and $I_{x}, I_{y}, I_{z}$ are the moment of inertias of the system around each axes. These equations are nonlinear, so they do not have an analytical closed-form solution. However, they can be solved under some relieving conditions.

Quaternion is used for the attitude representation herein. Therefore, the derivatives of the Euler parameters can be updated by using the kinematics equation as follows [15].

$$
\frac{d}{d t} q=\frac{1}{2}\left[\Omega^{\prime}\right] q
$$

Where $q$ is the attitude quaternion that represents the attitude of the satellite relative to the local-vertical-localhorizontal coordinate system and $\left[\Omega^{\prime}\right]$ is the skew symmetric matrix defined as follows.

$$
\left[\Omega^{\prime}\right]=\left[\begin{array}{cccc}
0 & \omega_{z} & -\omega_{y} & \omega_{x} \\
-\omega_{z} & 0 & \omega_{x} & \omega_{y} \\
\omega_{y} & -\omega_{x} & 0 & \omega_{z} \\
-\omega_{x} & -\omega_{y} & -\omega_{z} & 0
\end{array}\right]
$$

Also, the derivation of Euler angles from the attitude quaternion error is as follows.

$$
\left[\begin{array}{l}
\phi \\
\theta \\
\psi
\end{array}\right]=\left[\begin{array}{c}
\tan ^{-1}\left(\frac{2\left(q_{1} q_{4}+q_{2} q_{3}\right)}{1-2\left(q_{1}^{2}+q_{2}^{2}\right)}\right) \\
\tan ^{-1}\left(2\left(q_{4} q_{2}-q_{3} q_{1}\right)\right) \\
\tan ^{-1}\left(\frac{2\left(q_{4} q_{3}+q_{1} q_{2}\right)}{1-2\left(q_{2}^{2}+q_{3}^{2}\right)}\right)
\end{array}\right]
$$

The control law for stabilizing and attitude-maneuvering may be stated as a PD controller. Therefore the control torques will be as follows.

$$
\left\{\begin{array}{l}
T_{c x}=k_{x}\left(\phi_{c o m m}-\phi\right)+k_{d x} \dot{\phi} \\
T_{c y}=k_{y}\left(\theta_{c o m m}-\theta\right)+k_{d y} \dot{\theta} \\
T_{c z}=k_{z}\left(\psi_{c o m m}-\psi\right)+k_{d z} \dot{\psi}
\end{array}\right.
$$

Where $\phi_{\text {comm }}, \theta_{\text {comm }}$ and $\psi_{\text {comm }}$ are the Euler commands. $\dot{\phi}$, $\dot{\theta}$ and $\dot{\psi}$ are the Euler angular rates. Designing such a second-

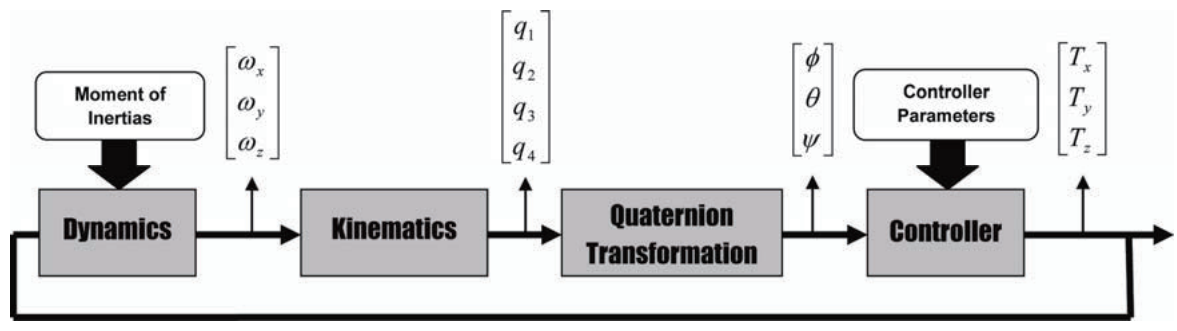

Fig. 3. Attitude control system block diagram 
order control system is a trivial automatic control problem. It is necessary to determine the controller coefficients so that the three one-axis control systems about the body axes have the desired dynamic characteristics, such as natural frequency and damping coefficients, which will preferably be equal for all axes. The overall simulation block diagram of the attitude control system is depicted in Fig. 3.

\section{Simulation of Pyramidal Reaction Wheel Arrangement}

The four reaction wheels are mechanized in a design frame, whose angular momentums form a vector $h_{w}=\left[h_{1} h_{2} h_{3}\right.$ $\left.h_{4}\right]^{T}$ as depicted in Fig. 4 .

Each reaction wheel accelerates or decelerates to generate different magnitudes of angular momentum. Regarding Fig. 4, the control torques acting on each axis can be stated as follows.

$$
\left[\begin{array}{l}
\hat{T}_{c x} \\
\hat{T}_{c y} \\
\hat{T}_{c z}
\end{array}\right]=\left[\begin{array}{c}
T_{c x} / \cos (\beta) \\
T_{c y} / \cos (\beta) \\
T_{c z} / \sin (\beta)
\end{array}\right]=\left[\begin{array}{cccc}
1 & 0 & -1 & 0 \\
0 & 1 & 0 & -1 \\
1 & 1 & 1 & 1
\end{array}\right]\left[\begin{array}{c}
T_{1} \\
T_{2} \\
T_{3} \\
T_{4}
\end{array}\right]
$$

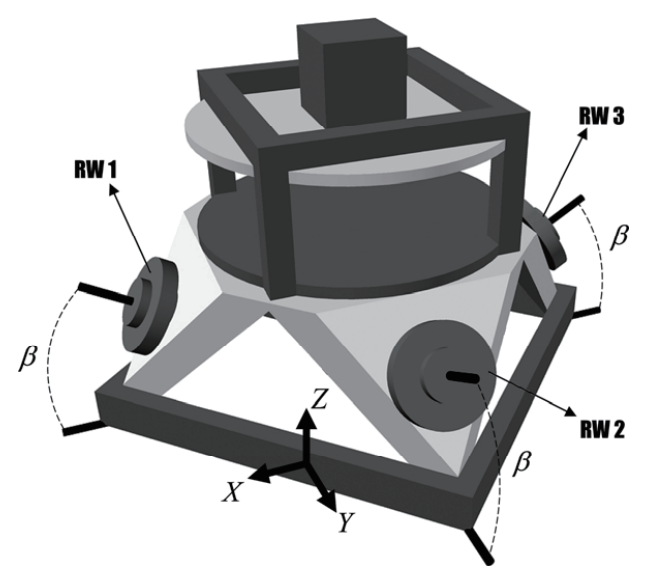

Fig. 4. Pyramidal reaction wheel arrangement
Here $\beta$ is the tilt angle of the wheel axes to the body plane. The control vector $\hat{T}_{c}$ is computed by the control law defined in equation (5).

To find the vector components of $T_{i}$ some optimizing criterion is assumed. The norm of the vector $T=\left[T_{1} T_{2} T_{3} T_{4}\right]^{T}$ is considered to be minimized as.

$$
J=\int T_{1}^{2}+T_{2}^{2}+T_{3}^{2}+T_{4}^{2} d t
$$

The Lagrangian will be.

$$
\begin{aligned}
& g=T_{1}^{2}+T_{2}^{2}+T_{3}^{2}+T_{4}^{2}+ \\
& p_{1}\left(T_{1}-T_{3}-\hat{T}_{c x}\right)+ \\
& p_{2}\left(T_{2}-T_{4}-\hat{T}_{c y}\right)+ \\
& p_{3}\left(T_{1}+T_{2}+T_{3}+T_{4}-\hat{T}_{c z}\right)
\end{aligned}
$$

The conditions for minimizing J will be.

$$
\frac{\partial g}{\partial T_{i}}=0
$$

Applying this condition yields the four wheels' command control torques as follows [16].

$$
\left[\begin{array}{c}
T_{1} \\
T_{2} \\
T_{3} \\
T_{4}
\end{array}\right]=\frac{1}{2}\left[\begin{array}{cccc}
1 & 0 & 1 / 2 & 1 / 2 \\
0 & 1 & 1 / 2 & -1 / 2 \\
-1 & 0 & 1 / 2 & 1 / 2 \\
0 & -1 & 1 / 2 & -1 / 2
\end{array}\right]\left[\begin{array}{c}
\hat{T}_{c x} \\
\hat{T}_{c y} \\
\hat{T}_{c z} \\
0
\end{array}\right]
$$

Consequently the changes of the wheels' rotational speed and power consumptions as functions of time can be obtained. The block diagram of this simulation is shown in Fig. 5.

By knowing the power consumption changes of the reaction wheels as a function of time, it is possible to determine the critical power consumption of the system regarding the attitude command.

Regarding the proposed analysis, the pyramidal reaction wheel arrangement optimal tilt angle can be obtained. The

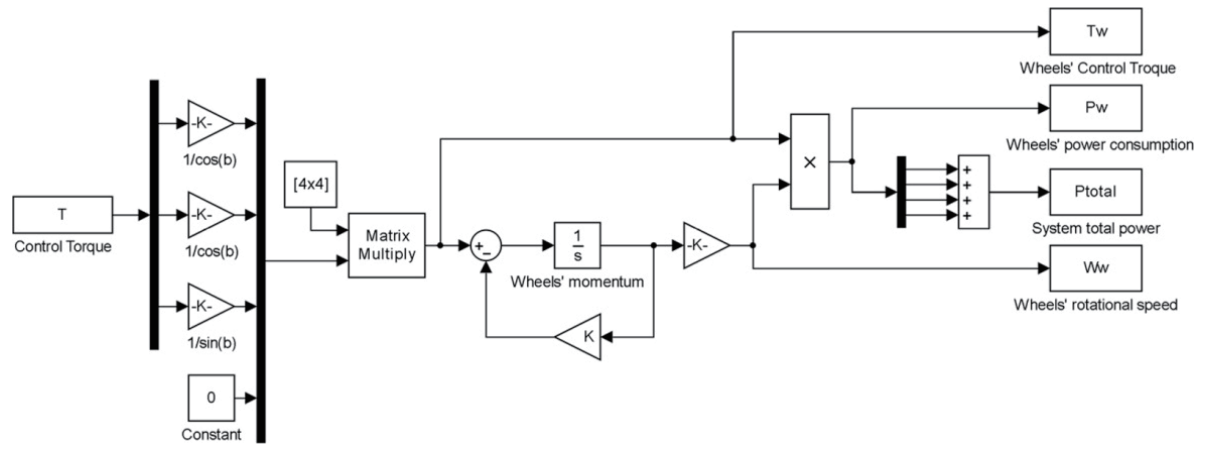

Fig. 5. System performance simulation block diagram 
input parameters including the moment of inertias of the system $\left[I_{x}, I_{y}, I_{z}\right]$, the attitude commands $\left[\phi_{\text {com }}, \theta_{\text {com }}, \psi_{\text {com }}\right]$ along with the initial conditions $\left[\phi_{0}, \theta_{0}, \psi_{0}\right]$ are known. Using the attitude control simulation based on equation (1), the coefficients for the controller can be specified according to equation (5) regarding the desired accuracy and settling time for the three Euler angles. If the wheels tilt angle is known, the reaction wheels' control torques required for the desired attitude maneuver will be achieved as shown in equation (6) and equation (10). Using a momentum management feedback loop, the rotational speed of the reaction wheels can be determined as shown in Fig. 5. Consequently, the power consumption of each reaction wheel can be achieved as a function of time during the attitude maneuver based on knowing the moment of inertias of the wheels. With this kind of simulation, the total power consumption of the system can be investigated and for each tilt angle.

\section{Optimization of Tilt Angle}

After the definition of the simulation process, the goal of the configuration design is to find the optimal tilt angle that minimizes the power consumption of the system. The inputs of the problem are considered based on the limitations and specifications of an attitude control simulator in the Space Research Laboratory as depicted in Fig. $6[17,18]$.

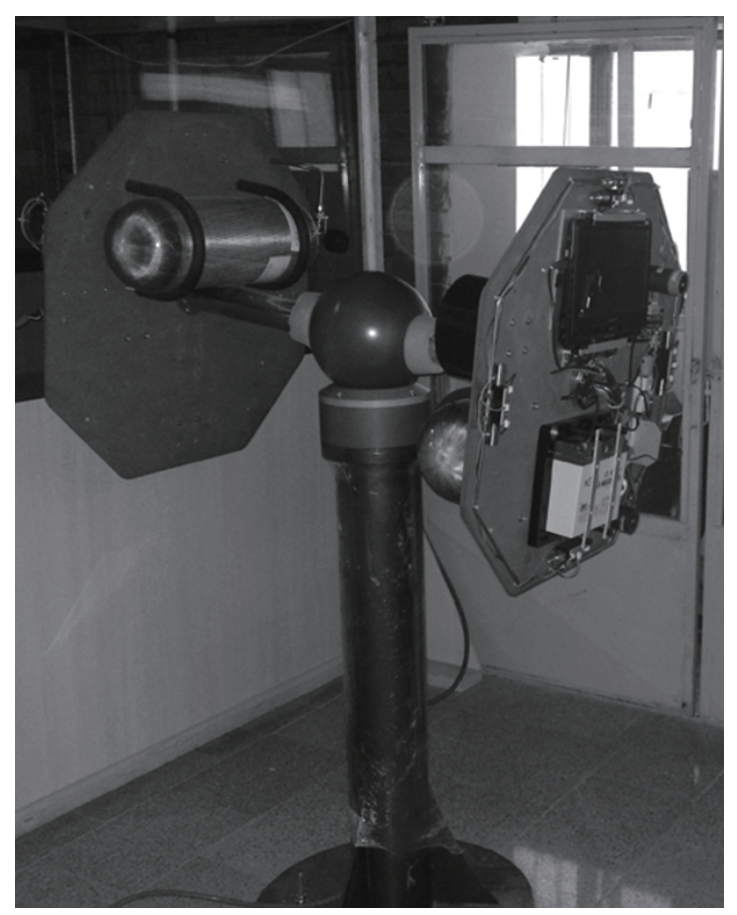

Fig. 6. The attitude control simulator in Space Research Laboratory
This three degree of freedom spacecraft simulator has been developed as part of a research program on spacecraft multi-body rotational dynamics and control, by the technical team in the Space Research Laboratory. This laboratorybased test-bed is used to explore various issues and concepts in spacecraft dynamics and control. Pyramidal reaction wheel arrangement will be implemented for the next version of this simulator. Therefore, the inputs are assumed based on the specifications of the spacecraft simulator. The moment of inertias of the system are assumed to be $\left[I_{x}, I_{y}\right.$, $\left.I_{z}\right]=[6,12,13] \mathrm{kg} \cdot \mathrm{m}^{2}$. For the desired 3D attitude maneuver, the attitude angle commands for the three axes are assumed to be $\left[\phi_{\text {com }}, \theta_{\text {com }}, \psi_{\text {com }}\right]=[30,20,-40] \mathrm{deg}$ and zero initial conditions are considered for simulation. The controller coefficients are determined as $k=[1.4,1.6,1.2]$ and $k_{d}=[-6$, $-5.8,-6.3]$ in order to have acceptable settling time and point accuracy for all axes. Euler angles response using the stated control law is shown in Fig. 7.

Data in Fig.7 was created by simulating Euler equations. This graph is based on the simulation block diagram depicted in Fig. 3 and is the target profile for tilt angle optimization.

As shown in Fig. 7, the controller is designed based on having acceptable angle gradients for all three axes. The settling time will be less than 30 seconds regarding the proposed control law. Based on this attitude maneuver, the control torques and the angular velocities about each axis will be as shown in Fig. 8 and Fig. 9.

After finalizing the control torques, the reaction wheels' performance can be simulated for different tilt angles. Each simulation reports a value for the power consumption of the system. Therefore, the changes of power consumption with different tilt angles can be plotted. In the selected case, the tilt angle is changed from 10 to 80 degrees with step of 1 degree. By considering the moment of inertia of $0.005 \mathrm{~kg}$.

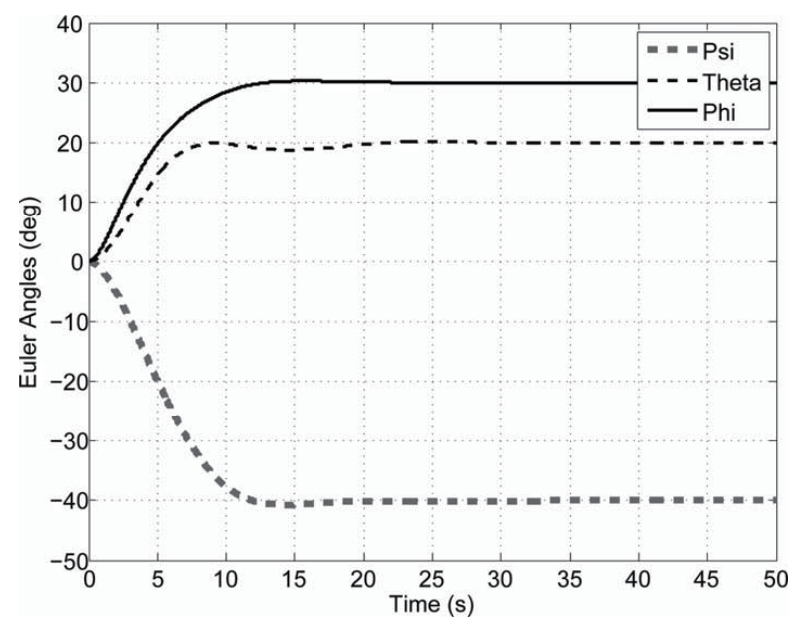

Fig. 7. System attitudes 
$\mathrm{m}^{2}$ for all the actuators, the reaction wheels' performance is simulated for each tilt angle. Results are illustrated in Fig. 10.

Figure 10 shows the power consumption of system for each tilt angle. According to this figure, it can be concluded that the tilt angle of 32 degrees produces the minimum power consumption; this tilt angle will be the best choice for the spacecraft simulator if the design criterion is power consumption. By simulating the reaction wheels'

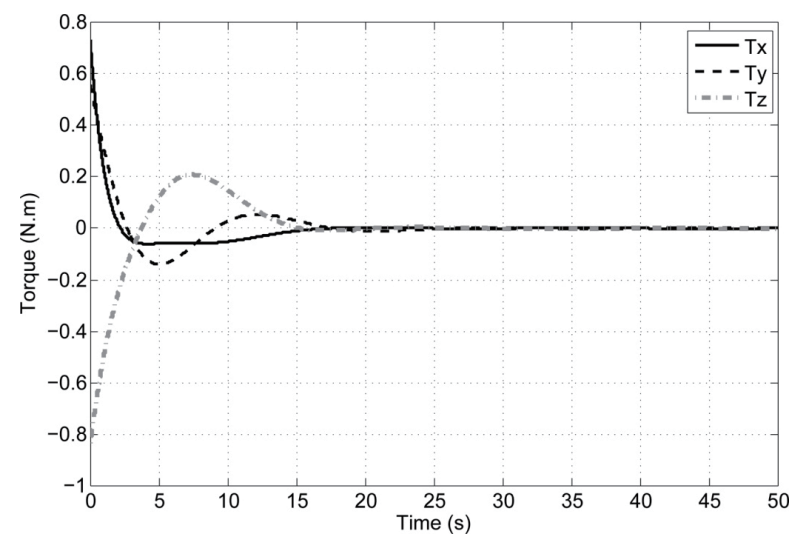

Fig. 8. System control torques

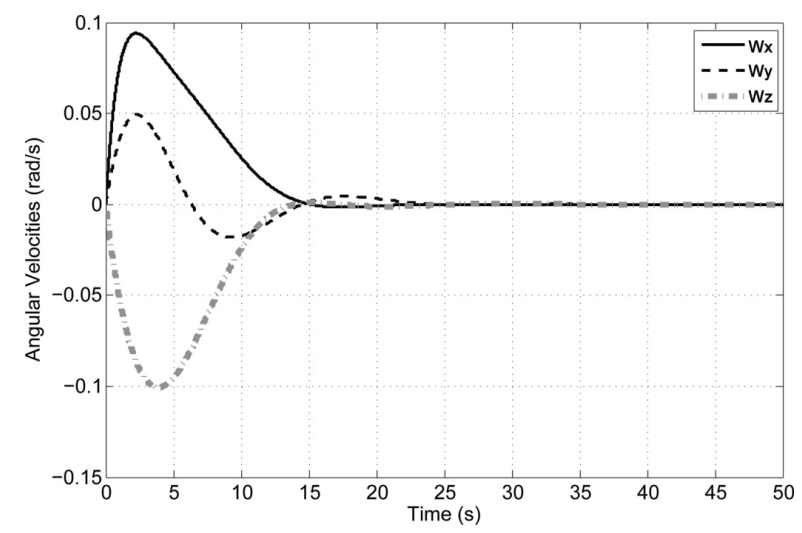

Fig. 9. System angular velocities

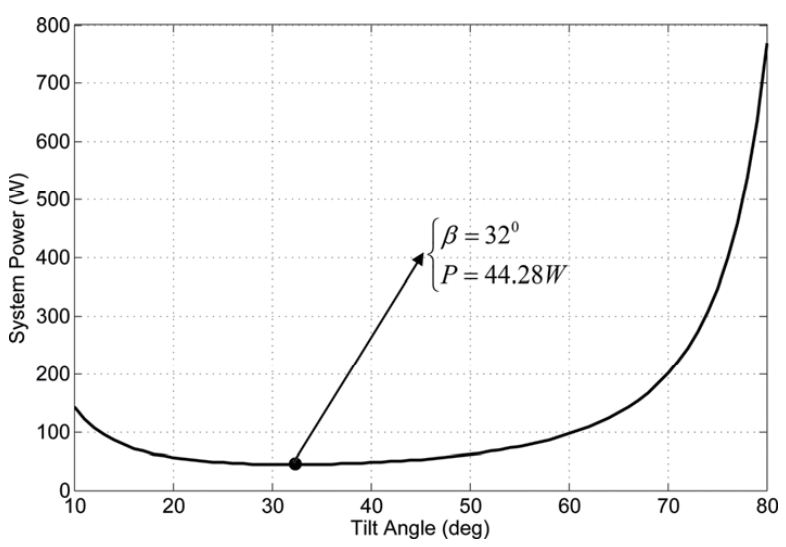

Fig. 10. System power consumption as a function of tilt angle performance with tilt angle of 32 degrees, the following results are achieved. Torque changes of reaction wheels along with control torques of three axes are given in Fig. 11.

The rotational speeds of the wheels are illustrated in Fig. 12.

Consequently, the power consumption of reaction wheels and total power consumption of the system are depicted in Fig. 13 and Fig. 14 respectively.

Clearly the maximum power consumption of the system is $44.28 \mathrm{~W}$ as specified in Fig. 10. The peak point of this plot is at the beginning of attitude maneuver at $t=0.77 \mathrm{sec}$. In this time, the system requires the power of 44.28 Watt. Results also show that the critical wheel in specifying the maximum control torque is not the same as the wheel in specifying the maximum rotational speed. The reaction wheel which produces highest control torque is RW3 and the maximum control torque reaches 0.6298 N.M in this attitude maneuver regarding Fig. 11. On the other hand, RW4 is the actuator which has the maximum rotational speed of 935 RPM at $t=2.19 \mathrm{sec}$ regarding Fig. 12. Note that the wheels in such systems are similar (having the same performance and

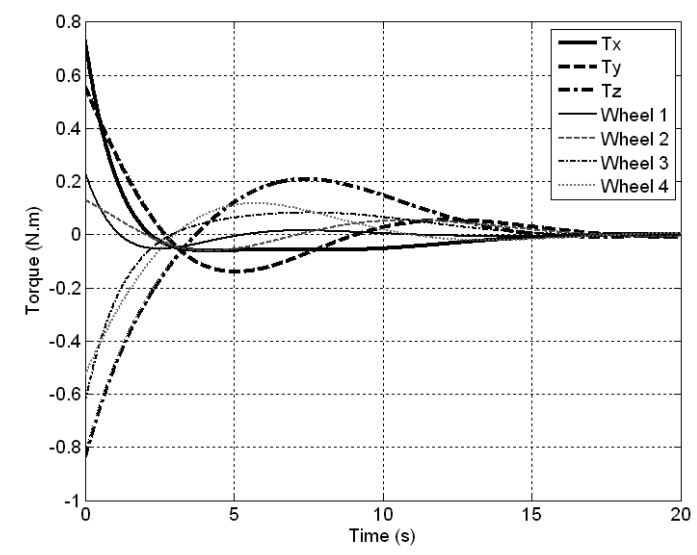

Fig. 11. Control torque of reaction wheels and three axes at a tilt angle of $32^{\circ}$

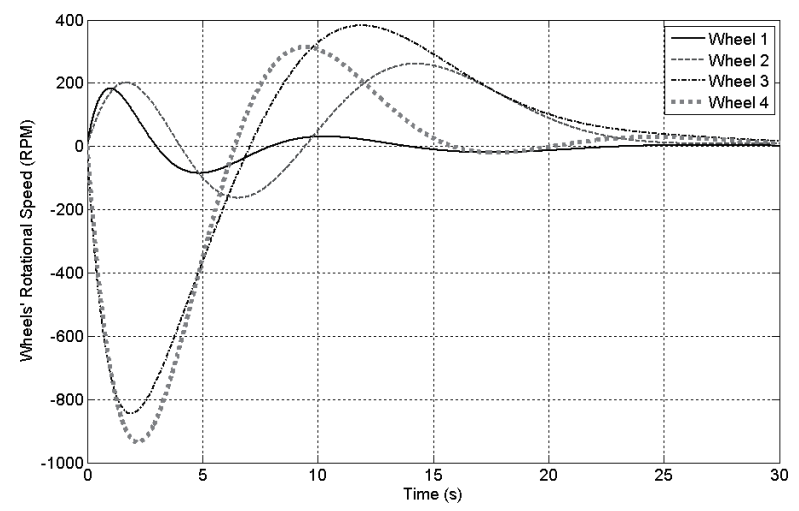

Fig. 12. Rotational speed of reaction wheels at a tilt angle of $32^{\circ}$ 
characteristics). Therefore, these values for torque and speed along with tilt angle should be considered in designing attitude control systems with reaction wheels in pyramidal arrangement.

The presented optimization indicates that the tilt angle of $32 \mathrm{deg}$ is the best choice for attitude control system considering the mentioned assumptions. The dependency of this value on the other parameters is an issue which should be considered. In the other word, the changes of optimal tilt angle with variations in the primary assumptions need to be investigated. In order to examine such dependencies, known and unknown design parameters should be specified.

The design process of attitude control systems can generally be divided into three phases: conceptual design, preliminary design, and final detailed design. The approach presented here is one of the aspects of the detailed design phase where some of the parameters are known and have been specified in previous design stages while some of them are still unknown and need to be specified along with tilt angle. Summary of these parameters are shown in Table 1.

While this approach is in the detail design phase, previous design stages have been completed and most of the

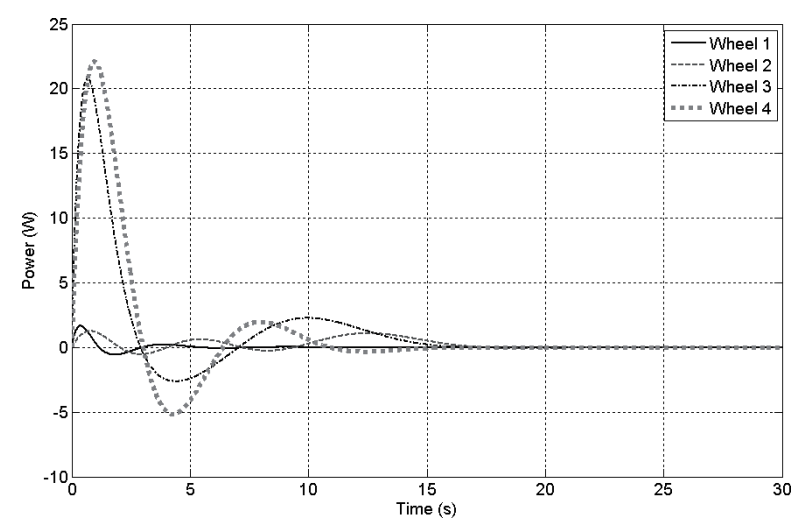

Fig. 13. Power consumption of reaction wheels at a tilt angle of $32^{\circ}$

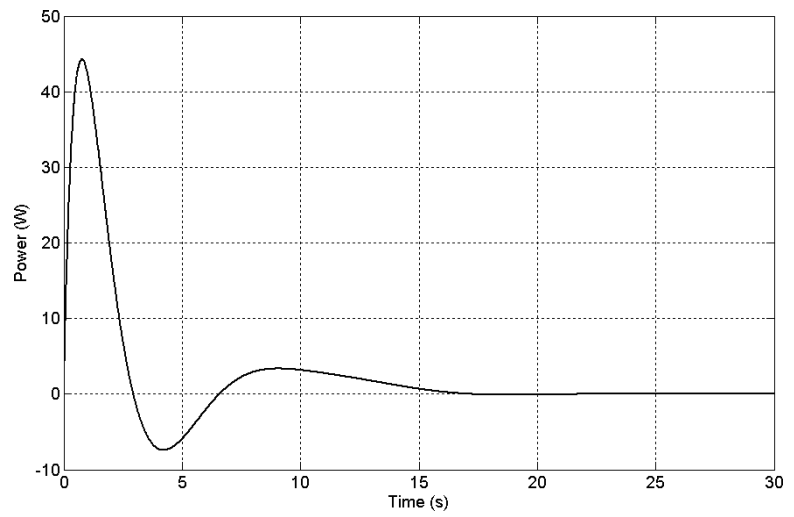

Fig. 14. System total power consumption at a tilt angle of $32^{\circ}$ parameters are known and fixed. The mission and objective is specified at the beginning of the entire design process so the desired attitude maneuver is known. Therefore parameters such as angle commands, settling time and initial conditions are known. Consequently the controller coefficients are

Table 1. Optimization parameters

\begin{tabular}{|c|c|c|}
\hline Parameter & Description & Assumed value \\
\hline$\left[I_{x}, I_{y}, I_{z}\right]$ & System's moment of inertias & {$[6,12,13] \mathrm{kg} \cdot \mathrm{m}^{2}$} \\
\hline$\left[\phi_{c o m}, \theta_{c o m}, \psi_{c o m}\right]$ & Attitude angle commands & {$[30,20,-40] \mathrm{deg}$} \\
\hline$[\phi(t), \theta(t), \psi(t)]$ & Desired attitude maneuver & Fig. 7 \\
\hline$\left[\phi_{0}, \theta_{0}, \psi_{0}\right]$ & Initial conditions & {$[0,0,0] \mathrm{deg}$} \\
\hline$t_{\text {total }}$ & Settling time & $30 \mathrm{sec}$ \\
\hline$\left[\begin{array}{l}k_{p} \\
k_{d}\end{array}\right]$ & PD controller coefficients & {$\left[\begin{array}{ccc}1.4 & 1.6 & 1.2 \\
-6 & -5.8 & -6.3\end{array}\right]$} \\
\hline$I_{w}$ & $\begin{array}{l}\text { Reaction wheels' moment of } \\
\text { inertias }\end{array}$ & $0.005 \mathrm{~kg} . \mathrm{m}^{2}$ \\
\hline
\end{tabular}

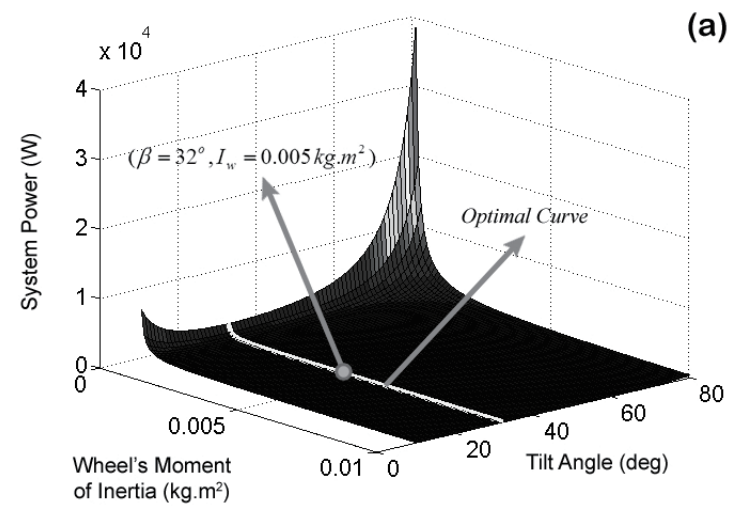

(a)

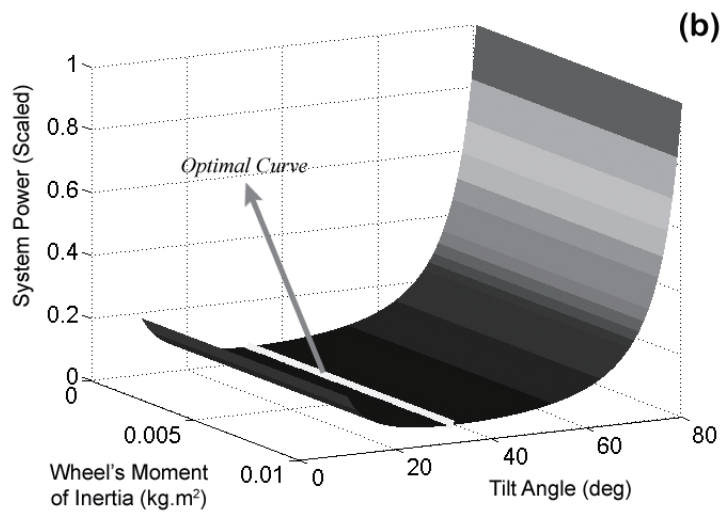

Fig. 15. System power as a function of tilt angle and wheel's moment of inertia 
known because all depend on the time profile of attitude angles. Primary design of the structure is also done and therefore the system's moments of inertias are known. In the current phase of design process, the only parameter which should be specified along with the tilt angle is the reaction wheels' moment of inertias. Tilt angle optimization along with changes in reaction wheels' moment of inertias are investigated in Fig. 15.

Fig. 15 shows the maximum power consumption of the system as a function of tilt angle and moment of inertia of the reaction wheel in pyramidal configuration. Fig.15 (a) shows the actual value of system power while Fig.15 (b) shows the scaled value of the surface between 0 and 1. Fig. 15 shows that it can be seen that the optimal values of tilt angle can be defined on a curve instead of a point (as in Fig. 10). This optimal curve represents the optimal values of tilt angle for each wheel's moment of inertia. It can be observed that the optimal tilt angle for each moment of inertia is the same, while system power gradients vary for each moment of inertia. Therefore, regardless of what moment of inertia the actuators have, the optimal tilt angle is $32 \mathrm{deg}$ for the system with moment of inertias of $[6,12,13] \mathrm{kg} \cdot \mathrm{m}^{2}$ and command angles of $[30,21,-40]$ deg.

\section{Conclusions}

The orientations of the four reaction wheels mechanized in a spacecraft involve a typical optimization of configuration design. This paper focused on optimization of pyramidal reaction wheel arrangement by finding the optimum tilt angle which results in minimum power consumption for the system.

By configuration optimization, the performances of the system can be maximized with minimum power consumption. Simulation shows that the tilt angle of 32 degrees in pyramidal reaction wheel arrangement results minimum power consumption for the assumed attitude control simulator with the moment of inertias of $\left[I_{x}, I_{y}, I_{z}\right]=[6$, $12,13] \mathrm{kg} \cdot \mathrm{m}^{2}$. This optimization process can be repeated for systems with different specifications and maneuverabilities.

Relatively little research has been conducted on optimization of a pyramidal arrangement. Similar researches by other authors concerning this kind of optimization indicate that the optimality criterion plays an important role in specifying the optimum tilt angle. While this paper focus on the minimization of power consumption, other design optimizations are based on optimizing the momentum/ torque envelop $[19,20]$ or workspace size [8]. Comparing the obtained optimal value for tilt angle in this study with the one used in similar simulations and projects indicates a small difference, leading us to conclude that the optimum tilt angle varies with requirements and criteria such as controller type, system moment of inertias, and desired maneuverability.

The problem of configuration optimization in pyramidal reaction wheel arrangements is a vast study and can be investigated with different approaches. Other criteria in designing this platform such as maximum control torque and maximum rotational speed of the wheels can also be considered in specifying the optimum tilt angle. It could be the optimal tilt angle of $32^{\circ}$ found in this study could be investigated to see if it also minimizes the critical control torque and the rotational speed of the wheels.

In an advanced approach, the tilt angle which minimizes all three criteria (power, torque, and rotational speed) could be analyzed and an optimum tilt angle can be found using proper weight functions for each criterion.

Another approach might be a sensitivity analysis of optimum tilt angle. Advanced performance simulation of this system may lead us to the relation of optimized tilt angle and other parameters such as the moment of inertias or the controller type.

\section{References}

[1] Dai, L. and Jin, G., "A 3-axis simulator for spacecraft attitude control research". IEEE International Conference on Information and Automation (ICIA). 2010, pp. 1040-1044.

DOI: 10.1109/ICINFA.2010.5512158

[2] Steyn, W., "A dual-wheel multi-mode spacecraft actuator for near-minimum-time large angle slew maneuvers", Aerospace Science and Technology, Vol. 12, No. 7, 2008, pp. 545-554.

DOI: $10.1016 /$ j.ast.2008.01.003

[3] Cruz, G. and Bernstein D., "Adaptive spacecraft attitude control with reaction wheel actuation". American Control Conference (ACC). 2013, pp. 4832-4837.

[4] Wisniewski, R. and Kulczycki P., "Slew maneuver control for spacecraft equipped with star camera and reaction wheels", Control engineering practice, Vol. 13, No. 3, 2005, pp. 349-356.

DOI: 10.1016/j.conengprac.2003.12.006

[5] Wang, B, Gong K, Yang D, and Li J., "Fine attitude control by reaction wheels using variable-structure controller". Acta Astronautica, Vol. 52 , No.8, 2003, pp. 61.-618.

DOI: 10.1016/S0094-5765(02)00133-9

[6] Ismail, Z. and Varatharajoo, R., "A study of reaction wheel configurations for a 3-axis satellite attitude control". Advances in Space Research, Vol. 45, No. 6, 2010, pp. 750- 
759.

DOI: 10.1016/j.asr.2009.11.004

[7] Guo, Y., Ma, G. and Li, C., "Steering law design to control moment gyroscopes for near minimum time attitude maneuver". The 5th IEEE Conference on Industrial Electronics and Applications (ICIEA). 2010, pp. 280-285.

DOI: 10.1109/ICIEA.2010.5516832

[8] Kurokawa H., Geometric Study of Single Gimbal Control Moment Gyros [Technical report]. Mechanical Engineering Laboratory, Agency of Industrial Technology and Science, Ministry of International Trade and Industry. 1998.

[9] Downs M., Adaptive Control Applied to the Cal Poly Spacecraft Attitude Dynamics Simulator [M.Sc thesis]. California Polytechnic State University. 2009.

[10] Silva S., Applied System Identification for a Four Wheel Reaction Wheel Platform [M.Sc thesis]. California Polytechnic State University. 2008.

[11] Logan J., Control and Sensor Development on a FourWheel Pyramidal Reaction Wheel Platform [M.Sc thesis]. California Polytechnic State University. 2008.

[12] Sarikan, A., Aydemir, M., Yavuzoglu, E. and Ozyurt, C., "Real time digital simulation of a satellite attitude control system". International Symposium on Power Electronics Electrical Drives Automation and Motion (SPEEDAM), 2010, pp. 827-832.

DOI: 10.1109/SPEEDAM.2010.5545123

[13] Zheng, S. and Han B. "Investigations of an integrated angular velocity measurement and attitude control system for spacecraft using magnetically suspended double-gimbal
CMGs". Advances in Space Research, Vol. 51, No. 12, 2013, pp. 2216-2228.

DOI: 10.1016/j.asr.2013.01.015

[14] Scholz, C., Romagnoli, D., Dachwald, B. and Theil, S., "Performance analysis of an attitude control system for solar sails using sliding masses". Advances in Space Research, Vol. 48, No. 11, 2011, pp. 1822-1835.

DOI: 10.1016/j.asr.2011.05.032

[15] Wie, B., Space vehicle dynamics and control. Reston, VA: American Institute of Aeronautics and Astronautics, 2008.

[16] Sidi, M., Spacecraft dynamics and control. 1st ed. Cambridge: Cambridge University Press; 1997.

[17] Mirshams, M., Taei, H. and Others., "A Systems Engineering Tool for Satellite Simulator Design". ASME 2010 10th Biennial Conference on Engineering Systems Design and Analysis. 2010; 5: 475-483.

DOI: 10.1115/ESDA2010-25341

[18] Mirshams, M., Taei, H., Novin Zadeh, A. and Ebadi, F., "A 3-DoF Satellite Simulator Design and Development". 60th International Astronautical Congress. 2009; D1 (6): 9.

[19] Shengyong, T., Jinjie, W, Kun, L. and Yulin Z., "Optimal configuration design of redundant flywheels and hardwarein-the-loop simulation". 31st Chinese Control Conference (CCC). 2012, pp. 4334-4338.

[20] Shengyong, T., Xibin, C., and Yulin, Z., "Configuration optimization of four dissimilar redundant flywheels with application to IPACS", 31st Chinese Control Conference (CCC). 2012, pp. 4664-4669. 